\title{
On the Use of Symmetry in Geometry.
}

\author{
By John W. Butrers, M.A., B.Sc,
}

Two features characterise the treatment of Geometry as presented in Euclid's "Elements": (1) the propositions are arranged in a definite sequence which cannot be greatly altered without invalidating the proofs ; (2) there are no methods of proof applicable to a large number of propositions. If we except the method of reductio ad absurdum, it is scarcely an exaggeration to say, for example, that in Book I no three propositions are proved by the same method.

Now that the order of Euclid has been abandoned it is desirable (1) that the proofs should be made as far as possible independent of the new order adopted, and (2) that methods having a wide application should be preferred to those suitable only for the proposition on hand.

It is the object of this paper to show that the general use of symmetry (in its various forms) would be of advantage.

The subject may be approached experimentally. Fold a sheet of paper along a line drawn on it and prick a number of holes through the double paper. Now unfold the paper. The holes (points) occur in pairs, one on each side of the line of fold (the axis of symmetry). The two points forming a pair are called corresponding points and, in general, lines, angles, triangles, etc., which coincide when the paper is folded (and are therefore congruent) are called corresponding lines, angles, triangles, etc.

The following propositions are obvious. (Fig. 6.)

1. Each point on the axis corresponds to itself, and, conversely, if a point corresponds to itself it is on the axis.

2. The axis corresponds to itself, as does every line perpendicular to the axis, and, conversely, if a line corresponds to itself it is an 
axis of symmetry or is perpendicular to an axis of symmetry. (Note: a figure may have more than one axis of symmetry.)

3. The line passing through two points corresponds to the line passing through the corresponding points, and, conversely, if two lines correspond they pass through corresponding points.

4. The distance between two points is equal to the distance between the corresponding points; conversely, if two points on two corresponding lines correspond, then if equal distances be measured from these points in the corresponding directions (both towards or both away from the axis) along these lines, the points thus found are corresponding points.

5. The angle made by a pair of lines is equal to the corresponding angle made by the corresponding lines, and conversely. (Note: if we consider the direction of a line towards the axis to be positive in all cases, then corresponding angles are of opposite sense.)

6. The distance of a line from a point is equal to the distance of the corresponding line from the corresponding point. (This assumes that only one perpendicular can be drawn from a point to a line.)

Many more similar propositions may be enunciated but the above are sufficient to show that the method is applicable to a large number of Euclid's propositions.

\section{Examples.}

I. 5. Given $\mathbf{A B}=\mathbf{A C}$, to prove that $\widehat{\mathbf{B}}=\widehat{\mathrm{C}}$. (Fig. 7.)

Draw AP, the bisector of $\widehat{A}$, as axis; this line may be called simply the axis of $\widehat{\mathbf{A}}$.

Since $\mathrm{AP}$ corresponds to $\mathrm{AP}$ (Prop. 2.) and $\mathrm{PA} B=\mathbf{P A} \mathrm{C}$, $\therefore \quad A B$ corresponds to AC. (Prop. 5 Converse.)

Since $A$ corresponds to $A$ and $A B=A C$,

$\therefore \quad$ B corresponds to $\mathrm{C}$; (Prop. 4 Converse.)

$\therefore \quad$ BC corresponds to CB ; (Prop. 3.)

$\therefore \quad \widehat{B}=\widehat{\text { C. }} \quad$ (Prop. 5.) 
I. 6. Given $\widehat{\mathrm{B}}=\widehat{\mathrm{C}}$, to prove that $\mathrm{BA}=\mathrm{CA}$. (Fig. 8.)

Draw $\mathrm{PQ}$, the perpendicular bisector of $\mathrm{BC}$; this line may be called the axis of $\mathrm{BC}$.

$\mathbf{P}$ corresponds to $\mathbf{P}$ and $\mathbf{P B}=\mathbf{P C}$;

$\therefore \quad$ B corresponds to C. (Prop. 4 Converse.)

$\mathbf{B}$ corresponds to $\mathbf{C}$ and $\widehat{\mathbf{B}}=\widehat{\mathrm{C}}$;

$\therefore$ BA corresponds to $\mathrm{CA}$; (Prop. 5 Converse.)

$\therefore \quad$ A corresponds to $A$.

$B$ corresponds to $\mathbf{C}, \mathbf{A}$ corresponds to $\mathbf{A}$ :

$\therefore \quad$ BA $=$ CA. (Prop. 4.)

I. 18. Give $\mathbf{A C}>\mathbf{A B}$, to prove $\widehat{B}>\widehat{C}$. (Fig. 9.)

Let the axis of $\widehat{A}$ meet $B C$ in $D$; then $B^{\prime}$, the point corresponding to $\mathrm{B}$, will lie in $\mathrm{AC}$, the line corresponding to $\mathrm{AB}$.

Since $A C>A B, B^{\prime}$ will lie between $A$ and $C$; hence

$\mathbf{A} \widehat{B}^{\prime} \mathrm{D}>\widehat{\mathrm{C}}$;

but $\widehat{\mathbf{B}}=\mathbf{A} \widehat{\mathbf{B}} ' \mathrm{D}$, these being corresponding angles;

$$
\therefore \hat{\mathrm{B}}>\widehat{\mathrm{C}} \text {. }
$$

I. 19. Given $\widehat{\mathrm{B}}>\widehat{\mathrm{C}}$, to prove $\mathrm{CA}>\mathrm{BA}$. (Fig. 9.)

Let the axis of $\widehat{A}$ meet $B C$ in $D$;

$$
\begin{aligned}
& \text { since } \widehat{B A D}+\widehat{B}+A \widehat{D B}=\widehat{D A C}+\widehat{C}+A \widehat{D C} \\
& \text { and } \widehat{B A D} \quad=\mathrm{D} \widehat{A} \mathrm{C} \\
& \text { also } \widehat{\mathbf{B}}>\widehat{\mathbf{C}} \text {; } \\
& \therefore \quad \mathbf{A D} B<\quad A \widehat{D} C \text {. }
\end{aligned}
$$

Let $\mathrm{DB}^{\prime}$ correspond to $\mathrm{DB}$,

then $\mathbf{A} \widehat{D} B^{\prime}=\mathbf{A} \widehat{D B}$ and $\therefore A \widehat{A D} B^{\prime}<\mathbf{A} \widehat{D C} ;$

$\therefore \quad B^{\prime}$ lies between $A$ and $C$;

$\therefore \quad \mathrm{AB}^{\prime}<\mathrm{AC}$ and $\therefore \mathrm{AB}<\mathrm{AC}$. 
I. 20. In the figure to the last proposition join $\mathrm{BB}^{\prime}$; then $\mathbf{D} \widehat{B}^{\prime} \mathbf{B}$ corresponds to $\mathbf{D} \widehat{\mathbf{B}} \mathbf{B}^{\prime}$;

$$
\begin{aligned}
& \therefore \quad \widehat{D B}^{\prime} \mathrm{B}=\mathrm{D}_{\mathbf{B B}}{ }^{\prime} \text {; } \\
& \text { hence } \widehat{C B}^{\prime} \mathbf{B}>\widehat{C} \widehat{B} B^{\prime} \text {; } \\
& \therefore \quad \mathrm{BC}>\mathrm{B}^{\prime} \mathrm{C} \text {; } \\
& \text { but } \quad \mathrm{AB}=\mathrm{AB}^{\prime} \text {; } \\
& \therefore \mathrm{AB}+\mathbf{B C}>\mathbf{A C} \text {. }
\end{aligned}
$$

(This proof supposes that $\mathrm{AB}<\mathrm{AC}$; if $\mathrm{AB} \nless \mathbf{A C}$, no proof is needed.)

Note: Since $\mathrm{BC}>\mathrm{B}^{\prime} \mathrm{C}$ and $\mathrm{B}, \mathrm{B}^{\prime}$ are corresponding points, we get the theorem : if lines be drawn from a point, not on the axis, to corresponding points, the one cutting the axis is greater than the other, and conversely.

I. 24. (Fig. 10.)

Let the triangles be $A B C, A B D$, having one pair of equal sides coinciding, and let $\widehat{B A D}$ be less than $\widehat{B A C}$. Then the axis of $\widehat{D A C}$ will fall within the larger angle $\mathrm{BAC}$ and will $\therefore$ cut $\mathrm{BC}$; but $\mathrm{C}$ and $\mathrm{D}$ are corresponding points ; $\therefore$ CB $>$ DB. (Theorem just proved.)

I. 25 may be proved as abore, using the converse of the theorem.

Otherwise : Draw PQ the axis of DC (Fig. 10.); then

$$
\begin{aligned}
\text { since } & \mathbf{A} \widehat{D C}=\mathbf{A C D}, \\
\therefore \quad & \mathbf{D A} \text { corresponds to } \mathrm{CA} ; \\
\therefore \quad & A \text { lies on } \mathbf{P Q} .
\end{aligned}
$$

Now $P Q$ cuts the greater line BC;

$$
\therefore \widehat{\mathbf{C A B}}>\mathbf{D A} \mathbf{A B} \text {. }
$$

These theorems have all been proved independently of the Congruence Theorems I. 4, 8, 26. These may be proved by the same method.

I. 4. (Fig. 11.)

Place the triangles so that they have a pair of equal sides $A B$ 
coincident and so that the other equal sides $A C, A C^{\prime}$ lie on opposite sides of $\mathrm{AB}$; then $\widehat{B A C}$ will be equal to $\mathrm{BAC}$.

$A B$ is an axis of symmetry of $A C, A C^{\prime}$, and since $A C=A C^{\prime}$,

$\therefore \mathbf{C}$ corresponds to $\mathrm{C}^{\prime}$ and hence $\mathrm{BC}$ corresponds to $\mathrm{BC}^{\prime}$;

$\therefore \mathrm{BC}=\mathrm{BC}^{\prime}$; also $\mathrm{A} \widehat{\mathrm{BC}}=\mathbf{A} \widehat{\mathrm{B}} \mathrm{C}^{\prime}$ and $\widehat{\mathrm{C}}=\widehat{\mathrm{C}^{\prime}}$.

\section{8. (Fig. 11.)}

In the triangles $\mathrm{ABC}, \mathrm{ABC}, \mathrm{AB}$ is common, $\mathrm{AC}=\mathrm{AC}^{\prime}$ and $\mathbf{B C}=\mathbf{B C}^{\prime}$.

$\mathrm{C}, \mathrm{C}^{\prime}$ lie on the circumference of a circle whose centre is $\mathrm{A}$ and which therefore has $A B$ as an axis of symmetry.

C, $\mathrm{C}^{\prime}$ lie also on the circumference of a circle whose centre is $\mathrm{B}$ and which therefore also has $A B$ as an axis of symmetry.

Hence $A B$ is an axis of symmetry of the whole figure.

Now the point corresponding to $C$ must lie on the circle $A$, since $C$ lies on that circle ; similarly it must iie on circle $B$; bence, since these circles have only one other point in common (viz. $\mathrm{C}^{\prime}$ ), $\mathrm{C}$ corresponds to $\mathbf{C}^{\prime}$;

$\therefore \mathrm{CA}$ corresponds to $\mathrm{C}^{\prime} \mathrm{A}$ and $\mathrm{BC}$ corresponds to $\mathrm{BC}^{\prime}$;

$\therefore \mathrm{CA} B=\mathrm{C}^{\prime} \widehat{A} \mathrm{~B}, \mathrm{CBA}=\mathrm{C}^{\prime} \widehat{B} A$ and $\widehat{\mathrm{C}}=\widehat{\mathrm{C}^{\prime}}$.

\section{26. (Fig. 11.)}

Place the equal sides of the triangles so that they coincide and take this line $\mathrm{AB}$ as axis.

Given $\mathbf{C A B}=\mathbf{C}^{\prime} \widehat{A} B ; \therefore \mathrm{CA}$ corresponds to $\mathbf{C}^{\prime} \mathbf{A}$;

also $\mathbf{A B C}=\mathbf{A} \widehat{\mathbf{B}} \mathbf{C}^{\prime} ; \therefore \mathbf{B C}$ corresponds to $\mathrm{BC}^{\prime}$;

$$
\therefore \mathrm{C} \text { corresponds to } \mathrm{C}^{\prime} \text {; }
$$

hence $\mathrm{CA}=\mathrm{C}^{\prime} \mathrm{A}, \mathrm{BC}=\mathrm{BC}^{\prime}$ and $\widehat{\mathrm{C}}=\widehat{\mathrm{C}^{\prime}}$.

When the given equal angles are $\mathrm{CAB}, \mathrm{O}^{\prime} \mathrm{AB}$ and $\mathrm{C}, \mathrm{C}^{\prime}$ we have $\widehat{C B A}=C^{\prime} B \widehat{A}$ by $I .32$ which may be proved without assuming I. 26.

For convenience, the propositions depending on Axial Symmetry have been grouped together, although this has necessitated the assumption of the truth of I. 16, I. 32 . 
Let us now consider Central Symmetry.

Suppose the line-segment AB (Fig. 12.) to rotate through two right angles about its middle point $C$, then $C A$ and $C B$ would change places, $A$ would take the place of $B$ and $B$ the place of $A$. Further, if $\widehat{C A D}=\widehat{C B E}$, then $A D$ and $B E$ would interchange places. Also, if $\mathrm{AD}=\mathrm{BE}$ then $\mathrm{D}$ and $\mathrm{E}$ would interchange places; and so on.

A figure which after a rotation through two right angles occupies the same position is said to have Central Symmetry, the centre of rotation being the centre of symmetry; parts which interchange positions are called corresponding parts.

The following propositions are obvious; they should be compared with the corresponding ones in Axial Symmetry. (Fig. 13.)

1. Each line passing through the centre corresponds to itself, and, conversely, if a line corresponds to itself it passes through the centre.

2. The centre corresponds to itself, and, conversely, if a point corresponds to itself it is the centre of symmetry.

3. The point of intersection of two lines corresponds to the point of intersection of the corresponding lines, and, conversely, if two points correspond, corresponding lines pass through them.

4. The angle between two lines is equal to the angle between the corresponding lines, and, conversely, if two lines correspond and at corresponding points on them equal angles are formed in the same direction (both clockwise or both counter-clockwise), then the other armas of these angles are corresponding lines.

5. The distance between two points is equal to the distance between the corresponding points, and conversely.

(Note: It will be shown that corresponding lines are parallel; corresponding distances are therefore of opposite sense.)

It will be noticed that for every statement made in axial symmetry there is a corresponding statement in central symmetry derived from it by the interchange of point for line and line for point. We thus get early an introduction to the principle of Duality. 
Examples of Central Symmetry.

I. 10̄. (Fig. 14.)

$\mathbf{E}$ is a centre of symmetry ;

EB corresponds to EA;

ED corresponds to EC;

$\therefore \quad \widehat{B E D}=\widehat{A E C}$.

(Prove $\mathrm{BEC}=\mathrm{AED}$ similarly).

I. 27. (Fig. 15.)

Given $\mathbf{A} \widehat{\mathbf{G}} \mathbf{H}=\mathbf{D} \widehat{\mathbf{H}} \mathbf{G}$.

Take $\mathbf{O}$ the mid-point of $\mathrm{GH}$ as centre.

OG corresponds to $\mathrm{OH}$; (Prop. 1.)

and since $\mathrm{OG}=\mathrm{OH}$,

$\therefore \quad$ G corresponds to $\mathrm{H}$; (Prop. 5 Converse.)

Because OGA $=\widehat{O H D}$,

$\therefore$ GA corresponds to HD, (Prop. 4.)

and consequently $\mathrm{HC}$ corresponds to GB.

Hence by Prop. 3 the point of intersection of GA and HC (if any) corresponds to the point of intersection of $\mathrm{HD}$ and $\mathrm{GB}$ (if any); i.e., if there is one point of intersection of $\mathrm{AB}$ and $\mathrm{CD}$, then there are two. As this is impossible, $A B$ and $C D$ have no point of intersection, i.e., they are parallel, i.e., corresponding lines are parallel.

I. 29. (Fig. 15.)

Given AB parallel to CD.

Take $O$ the mid-point of GH as centre of symmetry.

Then $H$ corresponds to $G$;

$\therefore$ the line corresponding to $\mathrm{CHD}$ passes through $\mathrm{G}$;

it is also parallel to CD (I. 27) and since there is only one parallel to $\mathrm{CD}$ through $\mathrm{G}$ (viz., $\mathrm{AB}$ ),

$\therefore \quad \mathrm{AB}$ corresponds to $\mathrm{CD}$;

$\therefore \quad \widehat{A G H}=\widehat{G H D}$.

Corollary. -If two lines are parallel and pass through corresponding points, the lines correspond. 
I. 33. (Fig. 16.)

Given $\mathrm{AB}$ equal and parallel to $\mathrm{CD}$.

Take the mid-point of $\mathrm{CB}$ as centre of symmetry.

$B$ corresponds to $\mathrm{C}$, and $\mathrm{BA}$ is parallel to $\mathrm{CD}$;

$\therefore \quad$ BA corresponds to CD.

Also $\mathrm{BA}=\mathrm{CD}$;

$\therefore \quad$ A corresponds to $\mathrm{D}$;

but $\mathbf{C}$ corresponds to $\mathbf{B}$;

$\therefore \mathrm{AC}$ corresponds to $\mathrm{DB}$;

$\therefore \quad \mathbf{A C}=\mathrm{BD}$ and $\mathrm{AC}$ is parallel to $\mathrm{BD}$.

I. 34. (Fig. 16.)

Given $\mathrm{AB}$ parallel to $\mathrm{CD}$ and $\mathrm{AC}$ parallel to $\mathrm{BD}$.

Take the same centre of symmetry as in $I$. 33 .

Because $\mathrm{B}$ corresponds to $\mathrm{C}$ and $\mathrm{BA}$ is parallel to $\mathrm{CD}$,

$\therefore \quad$ BA corresponds to CD.

Again $\mathrm{C}$ corresponds to $\mathrm{B}$ and $\mathrm{CA}$ is parallel to $\mathrm{DB}$,

$\therefore \quad$ CA corresponds to DB.

Hence $\mathrm{A}$, the intersection of $\mathrm{BA}, \mathrm{CA}$ corresponds to $\mathrm{D}$, the intersection of $\mathrm{CD}, \mathrm{BD}$;

$$
\therefore \quad \mathbf{A B}=\mathbf{C D}, \mathbf{A C}=\mathbf{B D}, \widehat{A}=\widehat{D} \text {, etc. }
$$

\section{Examples of application to problems.}

I. 9. (Fig. 17.)

Take $\mathrm{D}, \mathbf{D}^{\prime}$ and also $\mathbf{E}, \mathbf{E}^{\prime}$ pairs of corresponding points on $\mathbf{B A}$, BC. Let the corresponding lines $E D^{\prime}, E^{\prime} D$ meet in $F$. Then $F$ corresponds to itself; hence $\mathrm{F}$ lies on the axis; $\mathrm{BF}$ is the axis; $\therefore \mathbf{F B E}=\mathbf{F B} \mathbf{E}^{\prime}$.

I. 10. (Fig. 18.)

The above suggests the corresponding solution in central symmetry, viz.:- take $d, d^{\prime}$ and also $e, e^{\prime}$ pairs of corresponding lines through $\mathrm{R}$ and $\mathrm{S}$. Let the join of the points $e d^{\prime}, e^{\prime} d$ be $f$. Then $f$ corresponds to itself and therefore passes through the centre of symmetry, say $O$; hence $O R=O S$. 
In central symmetry the lines joining corresponding points pass through the centre and the points are equidistant from the centre. If the distances instead of being equal have a constant ratio, we get the theory of similar figures, which is beyond the scope of this paper.

In axial symmetry the lines joining corresponding points make equal angles with the axis and the points are equidistant from the axis. If the angles instead of being equal have a constant ratio (i.e., if the lines instead of being perpendicular to the axis make a constant angle with it), we get the theory of skew-symmetrical figures.

Let $A, A^{\prime} ; B, B^{\prime}$ (Fig. 19.) be pairs of corresponding points with reference to the skew axis $P Q$; i.e., $B P=P B^{\prime}, A Q=Q A^{\prime}$ and $A A^{\prime}$ is parallel to $\mathbf{B B}^{\prime}$.

Let the figure $A^{\prime} B^{\prime} P Q$ be rotated about $O$, the mid point of $P Q$, through two right angles. Then $\mathrm{QA}^{\prime}$ will lie along $\mathrm{PB}$ as $\mathrm{PA}^{\prime \prime}$; also $\mathbf{P B}^{\prime}$ will lie along $\mathrm{QA}$ as $\mathrm{QB}^{\prime \prime}$.

Since $\mathbf{P B}=\mathbf{Q B}$ " and $\mathbf{P A}^{\prime \prime}=\mathbf{Q A}$, $\therefore \mathrm{BB}^{\prime \prime}$ and $\mathrm{A}^{\prime \prime} \mathrm{A}$ are parallel to $\mathrm{PQ}$.

Hence $\mathrm{ABB}^{\prime \prime}=\mathrm{A}^{\prime \prime} \mathrm{B}^{\prime \prime} \mathrm{B}$, each being one half of the parallelogram $\mathrm{AA}^{\prime \prime} \mathrm{BB}^{\prime \prime}$;

to these equals add $B Q$ and we have

$$
A B P Q=A^{\prime \prime} B^{\prime \prime} Q P=A^{\prime} B^{\prime} P Q \text {. }
$$

Consider now two corresponding triangles $A B C, A^{\prime} B^{\prime} C^{\prime}$ (Fig. 20.), and let $\mathrm{AA}^{\prime}, \mathrm{BB}^{\prime}, \mathrm{CC}^{\prime}$ cut the axis in $\mathrm{P}, \mathrm{Q}, \mathrm{R}$.

Then the area of $\mathrm{ABC}$ is equal to the algebraic sum of the trapezia $A B Q P, B C R Q, C A P R$; and the area of $A^{\prime} B^{\prime} C^{\prime}$ equals the sum of the trapezia $A^{\prime} B^{\prime} Q P, B^{\prime} C^{\prime} R Q, C^{\prime} A^{\prime} P R$, those areas being reckoned positive when they lie to the left hand in going round in the order of the letters.

As the corresponding trapezia are equal but of opposite sign, the same is true of the triangles and hence of figures in general. Hence, in skew symmetry, corresponding areas are equal.

Euclid's theorems in areas follow at once.

I. 36. (Fig. 21.)

\section{Examples.}

Take as skew axis the line joining the mid points of DE and CF; then $\mathrm{ABCD}, \mathrm{HGFE}$ are corresponding areas and are . equal. 
I. 43. (Fig. 22.)

$\mathrm{EG}, \mathrm{BD}, \mathrm{HF}$ being diagonals of parallelograms, are bisected by $\mathrm{AKC}$ and these lines are parallel ; (Proof ?)

$\therefore A C$ is a skew axis and EBHK corresponds to GDFK;

$\therefore \mathrm{EBHK}=\mathrm{GDFK}$.

(The foregoing includes all the main propositions of Euclid's first book except I. 47 and its converse).

As another example take $\mathrm{AD}$, the median to $\mathrm{BC}$ as a skew axis (Fig. 23);

then $A B$ corresponds to $A C$;

$F$ the mid point of $A B$ corresponds to $E$ the mid point of $A C$;

$\therefore \mathrm{BE}$ corresponds to CF ;

hence they intersect on $\mathrm{AD}$; i.e., the medians are concurrent.

It is not the purpose of this paper to apply the method of symmetry beyond the subject matter of Euclid's first book. It is obvious, however, that it is of ready application to the properties of circles as developed in the third and fourth books.

In these days of the use of squared paper the transformation of a circle into an ellipse may form an appropriate conclusion :-

Draw a circle on squared paper and a line not perpendicular to the lines which cross the paper horizontally; take this line as skew axis (Fig. 24.).

Corresponding to each point of intersection of the circle with the series of horizontal lines, a point is taken on the opposite side of the skew axis and equidistant from it. By joining the series of points thus obtained we get an ellipse, and by counting the squares enclosed by each curve, the equality of the areas may be tested. Other properties may also be readily obtained; e.g., since the mid-points of the horizontal chords of the circle transform into the mid-points of the corresponding chords of the ellipse, the locus of these is a straight line. 